\title{
Long-term retention and overshadowing of proximal and distal cues following habituation in an object exploration task
}

\author{
Sarah Craig, Lorretto Cunningham, Lynda Kelly, Sean Commins* \\ Department of Psychology, National University of Ireland, Maynooth, Co. Kildare, Ireland
}

Received 1 December 2004; accepted 1 December 2004

\begin{abstract}
The object displacement task is a popular tool used to investigate spatial learning and memory. However, little attention has previously been given to long-term retention of spatial information following habituation to this task. Furthermore, the role of both proximal and distal cues in this type of passive retention of spatial information is controversial. In Study 1, we examined habituation in the object displacement task across 4 days and examined reactivity to spatial change 7 days post-acquisition. We found that rats habituated rapidly to the environment and retained this environment for the 7 days. Furthermore, this experiment shows that both proximal and distal spatial cues are important in the encoding of the environment during object displacement learning task. In Study 2, we examined the effect of overshadowing and demonstrate that proximal visual spatial cues can overshadow distal spatial cues if a conflict arises between both set.
\end{abstract}

(C) 2004 Elsevier B.V. All rights reserved.

Keywords: Spatial memory; Object displacement; Retention; Distal visual cues; Proximal visual cues; Overshadowing.

\section{Introduction}

Exploration is displayed by rats in the presence of novelty and enables them to acquire information about their environment, in particular spatial information. Although it can take many forms, exploration is most commonly seen as episodes of excited activity during which bouts of movement alternate with bouts of focused investigation (Eilam and Golani, 1989; Renner,

\footnotetext{
* Corresponding author. Tel.: +35317086182; fax: +3531 17084767 .

E-mail address: Sean.Commins@may.ie (S. Commins).
}

$1988,1990)$. When rodents are placed in an unfamiliar arena they display exploratory activity directed towards most aspects of this new environment, in particular towards the objects that are present (Granon et al., 1996). It is believed that an animal's spatial knowledge of its surroundings depends on such exploratory activity. Removal of an animal's opportunity to explore generally leads to poor spatial performance (Ellen et al., 1982, 1984; Chapius et al., 1987).

When the animal is placed in the same environment a second time there is a decrease in locomotor and exploratory activity (Poucet, 1989; Dai et al., 1995; Granon et al., 1996; Galani et al., 1998). This decrease 
in exploration (habituation) will continue to occur as long as the environment remains constant. A renewal of exploration is observed when there is some change in the environment, i.e. when items or places, which have not been experienced before, are encountered (O'Keefe and Nadel, 1978; Save et al., 1992; Galani et al., 1998). Furthermore, renewal of exploration after spatial rearrangement implies that the second arrangement is compared with some internal representation of the first arrangement and thus indicates the ability of the animal to retain spatial knowledge (Granon et al., 1996).

During exploration, it is thought that rodents build up a cognitive map or internal representation of the experimental arena (O'Keefe and Nadel, 1978). It is as yet unclear whether all aspects of this arena are important in the laying-down of the spatial memory. Do they associate cues outside the arena (distal visual cues) with the position of objects inside the arena? Are distal visual cues ignored and only proximal cues (those within the experimenting arena) retained? Very few studies have focused on the importance of these cues in passive learning tasks. Most experiments were undertaken using active learning tasks such as the Morris water maze, where strong associations between the distal cues and an escape platform have to be made in order for the rat to learn the task and removal or rearrangement of these distal cues leads to learning impairments (Rudy et al., 1987; Chapillion, 1999). Other active learning tasks work on the basis of reward: certain cues signal where a reward such as food will be found (Chapillion et al., 1995). However, the object displacement task (Poucet, 1989) is a task where animals are not clearly taught any relationship between the consequences of their behaviour and the presence of cues. So will the rats use these cues to build up a spatial representation of the arena? If so, which cues will be more important in this type of spatial learning? It is known that displacement of an object within the environment (proximal cue) leads to renewal of exploration, thereby suggesting these are important for learning the spatial arrangement of the environment and also that the arrangement has been retained (Thinus-Blanc et al., 1992). Similar results were found elsewhere (Delu et al., 1997), but Moses et al. (2002) found that rearrangement of proximal or distal cues was not enough to illicit impairments in spatial memory.

This conflict concerning the importance of proximal and distal visual cues is not confined to tasks such as the object exploration task (Vollmer-Conna and Lemon, 1998; Chapillion, 1999; Tropp and Markus, 2001). It is possible that the stability of each set of cues will determine which will be favoured (Biegler and Morris, 1996). In the radial arm maze, it was found that rats preferentially used distal cues when the proximal cues were not salient (Hunt and Aggleton, 1998). Carman and Mactutus (2002) found that animals trained in a Morris water maze with a stable visual cue did not rely on extramaze cues to learn the task, however animals trained where the proximal cues were not stable rely solely on distal cues. This overshadowing of intramaze cues by extramaze cues has also been documented by other experimenters (Diez-Chamizo et al., 1985; Chapillion, 1999).

\section{Study 1}

In the first study, we will make use of the object exploration task to test spatial memory in rats and examine the role of both proximal and distal visual cues in retaining the spatial layout of the environment. First, we will habituate the animals to the arena over 4 days with four trials per day. Following this, we will change the arrangement of one of the objects, within the arena (proximal cue) 7 days post-habituation, and hypothesise that rats in this condition will renew exploration around that object, demonstrating that spatial arrangement of proximal cues has been retained over a 7-day period. In the second condition, we will rotate the distal cues while keeping the proximal cues constant and hypothesise that the animals will increase exploration in general. Finally, we will relocate both proximal and distal cues to determine if one set of cues overshadows the other and hence is more important for this type of spatial learning.

\subsection{Method}

\subsubsection{Animals}

Male Wistar rats (200-300 g: Bioresources Unit, University of Dublin, Trinity College) aged approximately 3 months were used as subjects. Rats were housed three per cage and kept in a temperaturecontrolled room, which was maintained on a fixed light-dark cycle. The rats were given free access to food and drink. All testing was carried out during the 
light phase and all rats were well-handled before experimentation.

\subsubsection{Apparatus}

The apparatus consisted of a black circular fibreglass arena $($ diameter $=130 \mathrm{~cm}$; height $=38 \mathrm{~cm})$ resting on a table $70 \mathrm{~cm}$ above the ground. Curtains surrounded the entire arena at a distance of $50 \mathrm{~cm}$ from apparatus. Distal visual cues surrounded the arena and included a black poster $(55 \mathrm{~cm} \times 81 \mathrm{~cm})$ on the eastern wall, a lamp with a red bulb $(60 \mathrm{~W})$ on the northeast corner and a lamp with a blue bulb $(60 \mathrm{~W})$ on the northwest corner. The experimenter, wearing a white laboratory coat stood at the southern side of the arena during testing. Four objects were placed in a square formation at the centre of the arena approximately $40 \mathrm{~cm}$ apart. The four objects included a rectangular plastic box (height $=13.5 \mathrm{~cm}$; length $=27 \mathrm{~cm}$; width $=11 \mathrm{~cm}$ ), a concrete pillar $($ height $=18.5 \mathrm{~cm}$; diameter $=12.5 \mathrm{~cm}$ ), a wooden tree stump (height $=12 \mathrm{~cm}$; diameter $=8 \mathrm{~cm}$ ) and a plant in a glass vase (height $=33 \mathrm{~cm}$; diameter $=10.5 \mathrm{~cm}$ ).

\subsubsection{Procedure}

All rats were placed in the centre of the arena and were given four trials per day for 4 consecutive days to explore the arena. Each trial lasted $1 \mathrm{~min}$ and the inter-trial interval was approximately $15 \mathrm{~s}$.

To assess exploratory behaviour the experimenter recorded the number of nose contacts each rat made with the individual objects. This did not include bumping or backing into the objects, or rearing. One contact was counted each time the animal made nose contact with the object of investigation. If the animal continued to sniff the object, after the initial contact, without withdrawing contact this continued to be counted as one nose contact. Following habituation all animals were returned to their home cages.

Retention was assessed 7 days after the end of habituation and the rats were randomly assigned to one of the four groups. The retention trials lasted $1 \mathrm{~min}$ as this was the length of trials during the habituation phase. It is also possible that habituation would re-occur within the trial if it were to last any longer.

The control group $(n=7)$ was allowed to explore the arena for $1 \mathrm{~min}$ where no changes had been made to the arena or to the distal visual cues.
The object displacement group $(n=8)$ was placed in the centre of the arena for 1 min where the wooden object was moved toward the side of the arena at a distance of $15 \mathrm{~cm}$ from the periphery. None of the distal visual cues were changed.

The cue rotation group $(n=7)$ was placed in the centre of the arena and once again allowed to explore for $1 \mathrm{~min}$. No changes were made to the arena however the distal cues on the walls were rotated $180^{\circ}$ clockwise so that the black poster was now affixed to the west wall, the lamp with the red bulb was affixed to the southwest corner and the lamp with the blue bulb to the southeast corner and the experimenter stood at the northern end of the arena.

The cue rotation and object displacement group $(n=8)$ were also placed for $1 \mathrm{~min}$ in the arena where the cues were rotated $180^{\circ}$ clockwise. In addition, the wooden block was also moved toward the side of the arena.

To eliminate the possible biasing of any olfactory cues, the experimenter handled every object.

\subsubsection{Statistics}

A series of repeated and/or mixed ANOVAs were used. Where appropriate independent and/or dependent $t$-tests were carried out using SPSS (Version 10) $\left({ }^{*} P<0.05 ;{ }^{* *} P<0.01\right)$.

\subsection{Results}

\subsubsection{Habituation}

All animals habituated rapidly to their environment over the 4 days (see Fig. 1a). The mean number of nose contacts made with the four objects decreased from $25.03 \pm 0.93$ on day 1 to $12.43 \pm 0.77$ on day 4 . The habituation was confirmed by a one-way ANOVA. An overall significant difference $(F=15.96$, d.f. $=3,476$, $P<0.001)$ was found across the four days. A series of post-hoc tests (Tukey, $P<0.05$ ) confirmed that the number of nose contacts on day 1 was significantly higher compared to the other 3 days. No other significant differences were noted.

\subsubsection{Retention}

Reaction to spatial novelty and/or environmental manipulation was assessed 7 days post-habituation. Fig. 1b shows the mean number of nose contacts made with the objects, on the retention trial compared 

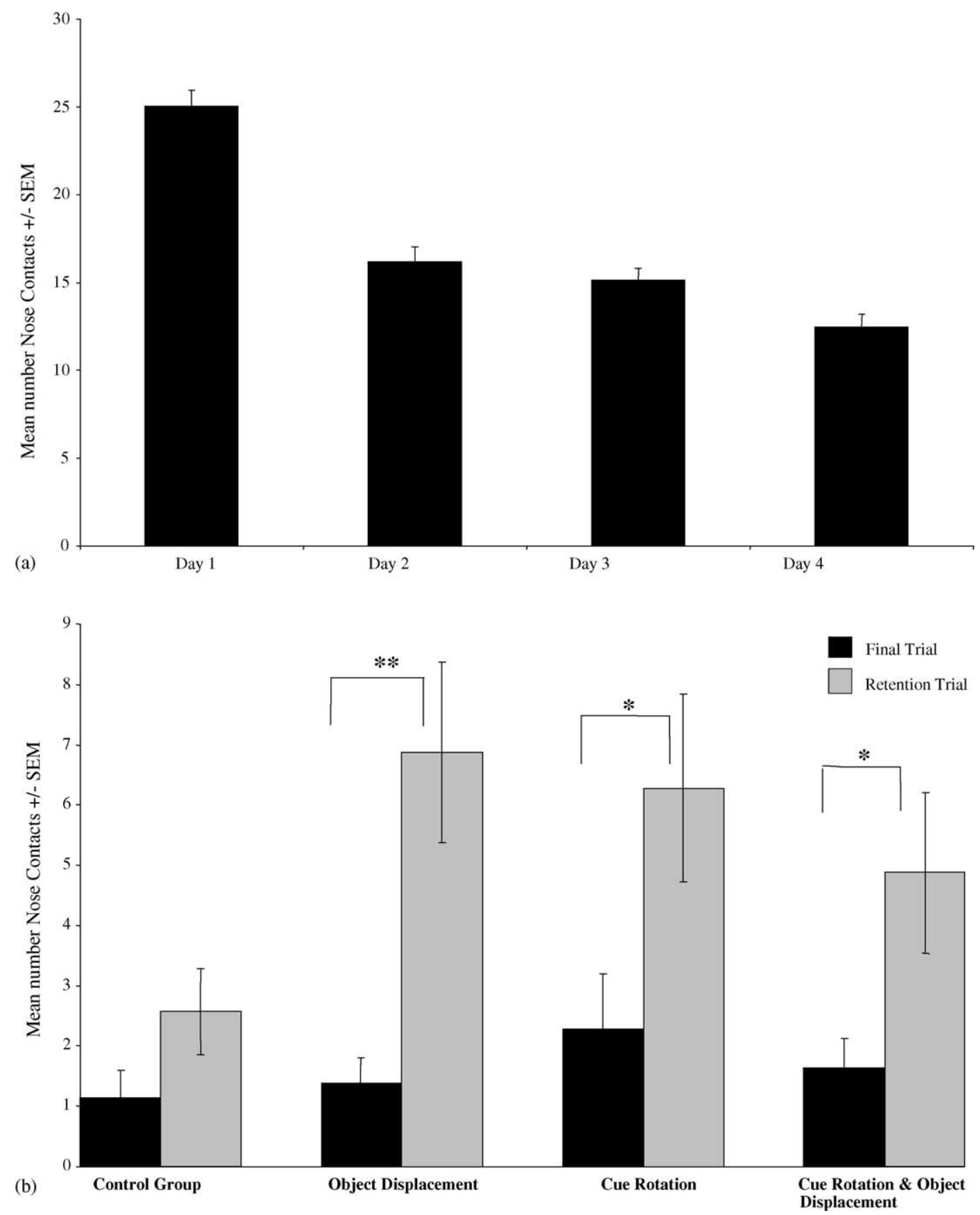

Fig. 1. (a) Mean number of nose contacts made with all objects over the 4 days of habituation. (b) The mean number of nose contacts made with all the objects on the last trial of the last day of habituation and on the retention trial for the four groups (control group; object displacement group; cue-rotation group; cue-rotation and object displacement group). 
with the mean number of nose contacts on the final trial of the final day of habituation for each of the four experimental groups. A two-way repeated ANOVA was conducted with trial (final trial versus retention trial) used as the within-group measure and groups (control; object displacement; cue-rotation; cue-rotation and object displacement) as the betweengroup measure. There was an overall significant effect for trial $(F=22.85$, d.f. $=1,26, P<0.01)$. There was also a significant effect for group $(F=3.92$, d.f. $=3,26$, $P<0.05)$ and an interaction effect $(F=4.92$, d.f. $=3,26$, $P<0.01$ ).

Subsequent analysis, using $t$-tests, for each of the groups was conducted. No significant change was observed in the total number of nose contacts with the objects during the retention trial compared with the final trial of habituation (see Fig. 1b, first panel: $t=-2.08$, d.f. $=7, P>0.05$ ) for the control group. A significant increase in the total number of nose contacts with the objects on the retention trial compared with the final trial of habituation was observed (see Fig. 1b, second panel: $t=-3.64$, d.f. $=7, P<0.01$ ) for the object displacement group. There was a significant increase in the total number of nose contacts made with the objects in the cue-rotated group on the retention trial when compared to the final trial of habituation (see Fig. 1b, third panel: $t=-2.52$, d.f. $=6, P<0.05)$. There was a significant increase in the total number of nose contacts with the objects during the retention trial when compared with the final trial of habituation (see Fig. 1b, fourth panel: $t=-2.44$, d.f. $=7, P<0.05$ ) for the cue-rotation, object displacement group.

We then assessed each individual group (using repeated-measures ANOVA) on the number of nose contacts with each individual object on the retention trial compared to the number of nose contacts on the last trial of habituation. Object type (wood, plant, box and pillar) was used as the between-group measure and trial (final trial versus retention trial) as the within group measures.

Fig. 2a demonstrates that no overall significant effect for trial was observed $(F=2.33$, d.f. $=1,24$, $P>0.05)$. There was also no significant effect for object $(F=2.14$, d.f. $=3,24, P>0.05)$ or interaction effect $(F=0.77$, d.f. $=3,24, P>0.05)$ for the control group.

For the object displacement group (Fig. 2b) an overall significant effect for trial was observed $(F=19.99$, d.f. $=1,28, P<0.001)$. There was also an overall significant effect for object $(F=10.45$, d.f. $=3,28$, $P<0.001)$ and an interaction effect $(F=14.4$, d.f. $=3$, $28, P<0.001)$. Further analysis using multiple paired $t$-tests revealed a significant increase in the number of nose contacts, on the retention trial, with the displaced object (wooden block) $(t=-4.63$, d.f. $=7$, $P<0.01)$ and with the concrete pillar $(t=-3.24$, d.f. $=7, P<0.05)$ compared to the final trial of habituation. No other increases were noted.

For the cue-rotation group (Fig. 2c) an overall significant effect for trial $(F=9.45$, d.f. $=1,24, P<0.01)$ was observed. There was no significant effect for object $(F=0.12$, d.f. $=3,24, P>0.05)$ and there was also no interaction effect $(F=0.32$, d.f. $=3,24, P>0.05)$.

Finally, for the cue-rotation and object displacement group (Fig. 2d) an overall significant effect for trial $(F=8.73$, d.f. $=1,28, P<0.01)$ was noted. A significant effect for object $(F=3.02$, d.f. $=3,28, P<0.05)$ but a non-significant interaction effect between object and trial $(F=0.15$, d.f. $=3,28, P>0.05)$ was observed.

\subsection{Discussion}

We demonstrate that displacement of an object within the arena leads to both a general increase in exploration and an increase in exploratory behaviour involving the proximally displaced object. It has been well documented that an animal renews its interest in a spatially changed object (Save et al., 1992; Dix and Aggleton, 1999; Save and Poucet, 2000). This renewal of exploration after spatial rearrangement implies that the second arrangement is compared with some internal representation of the first arrangement and indicates the ability of the animal to retain spatial knowledge of the proximal environment (Granon et al., 1996). Most experiments involving the object exploration task examine the reactivity to either a spatial change or an object change immediately after habituation. Gemmell and O'Mara (1999) and Galani et al. (1998) for example, allowed four trials of habituation with a 3-min inter-trial-interval and displaced the object on the fifth trial. Therefore, retention of spatial knowledge following the displacement most likely relies heavily on spatial working memory, similar to the habituation process itself (Ennaceur and Delacour, 1988). We suggest that knowledge of the spatial arrangement of proximal cues acquired passively can also be retained 

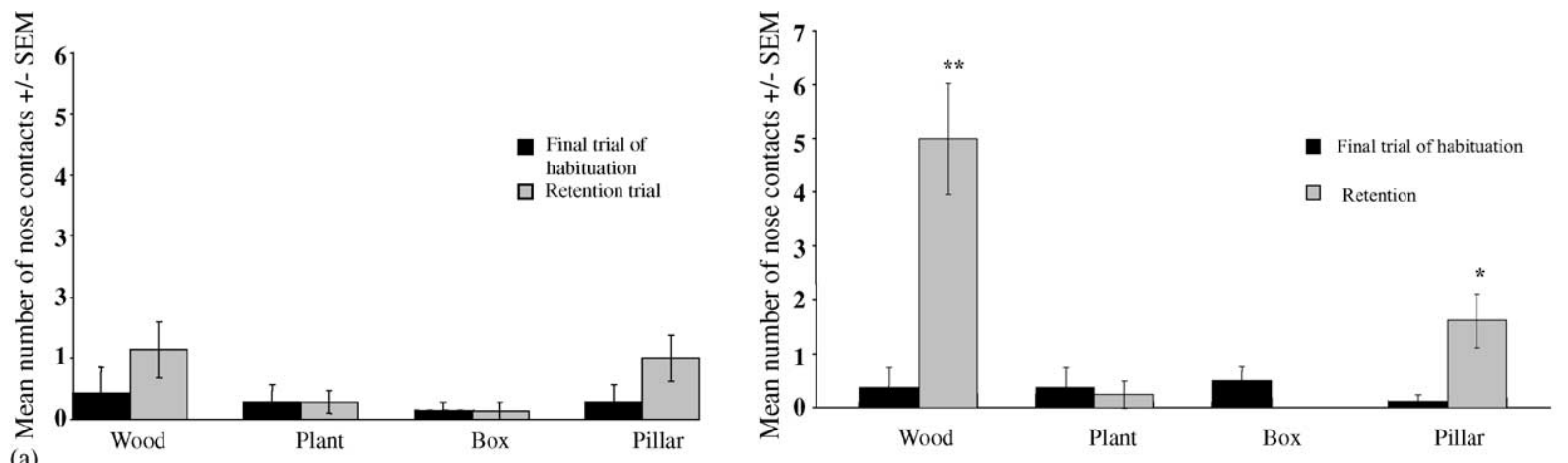

(b) (Displaced object)
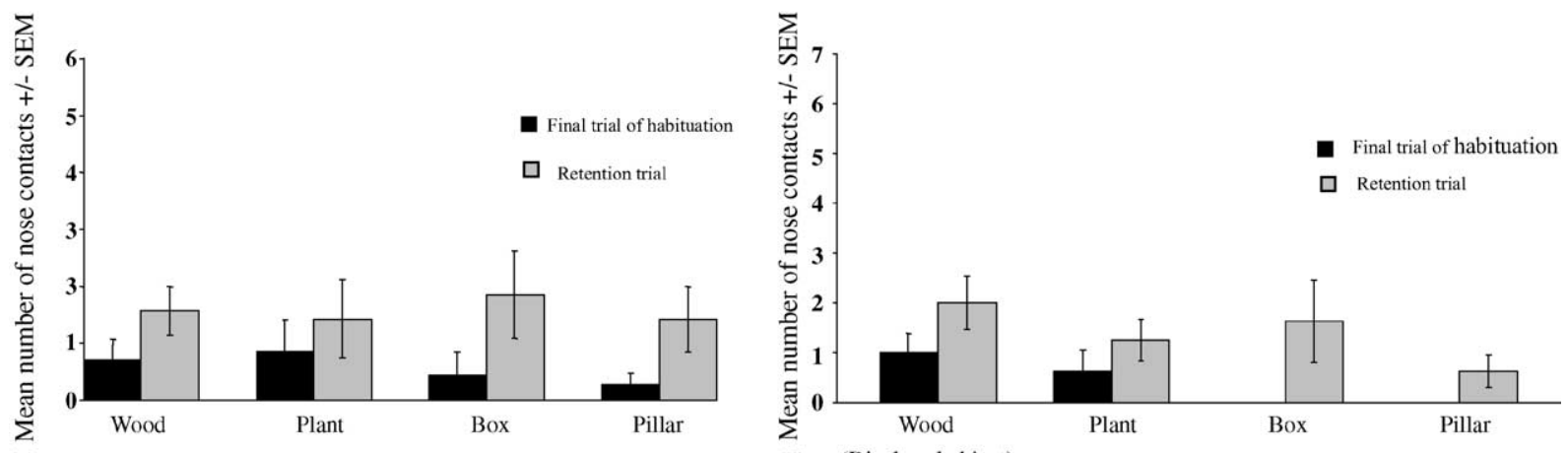

(c)

(d) (Displaced object)

Fig. 2. The mean number of nose contacts made with each of the four objects on the last trial of the last day of habituation and on the retention trial for the control group (a), the object displacement group (b), cue-rotation group (c) and the cue-rotation and object displacement group (d).

in long-term memory (at least for 7 days). The strength of the long-term retention may be partially due to the habituation procedure that was employed in the present experiment (see also Commins et al., 2003). We allowed animals to habituate to the environment within and across a number of days and found that exploratory activity decreases across each day. We find no significant difference in exploration on days 2-4 suggesting a rapid encoding of the environment (within the first day).

We also demonstrate that distal cues play a role in the retention of an environment that has been passively acquired. Rotation of the distal cues $180^{\circ}$ led to a general increase in exploratory behaviour. This suggests that the spatial arrangement of the distal cues was retained for at least 7 days. In active learning tasks (for example the radial arm maze or water maze), animals have the ability to retain information over a long period of time. Bolhuis et al. (1994) and
Mumby et al. (1999) for example demonstrate that in the water maze task a rat's memory for the location of the platform is retained for up to 14 weeks. While Van Groen et al. (2001) demonstrate retention of the task following 12 months. The ability of animals to retain such spatial information has been shown to rely on distal cues (Chapillion, 1999; Mc Gauran et al., 2004). The role of distal cues in retention in a task that has been passively acquired; such as the object displacement task is controversial. Moses et al. (2002) were unable to determine a role for either proximal or more importantly distal cues in spatial memory. While Dix and Aggleton (1999) suggest that animals can link surrounding cues as they explore the environment. This experiment shows that although the distal cues may not be essential in providing information about the arena (as would be the case where animals learn the escape platform or the location of food relative to the distal cues) distal cues are 
however encoded and subsequently retained for at least a week.

We attempted to examine the effects of overshadowing of one set of cues by the other in this study. Although we found that the cue-rotation and object displacement group did increase their reactivity to the objects 7 days post-acquisition, we were unable to determine directly whether overshadowing occurred on the retention trial due to the nonsignificant interaction effect. We decided therefore to examine the issue of overshadowing in a second set of experiments.

\section{Study 2}

One issue that was not addressed in the first study is the nature of the distal visual cues being presented. Distal cues must be fairly large and obvious to the animal as it is known that the visual acuity of albino rats is poor and they are thought to be fairly short-sighted (Robinson et al., 2001). We were confident that the visual distal cues in the first study were large enough for the animals to notice, as there was an increase in reactivity following rotation of the distal cues alone. Furthermore, we have used this exact set-up in previous experiments both in the object exploration and the water maze task (for example see Commins et al., 2003). It is known that both a spatial manipulation of an object already present in the arena or a replacement of an object with a novel one (without manipulation) elicits an increase in exploration (Galani et al., 1998). To ensure that the distal visual cues were noticed in order that we could test the effects of overshadowing directly, we also spatially manipulated the distal cues for the retention trial (cue-rotation). In addition, we replaced the distal cues used during training with novel ones during the retention trial (cue replacement). If overshadowing of the proximal cues by the distal cues were to occur we suggest that animals will display extra reactivity in both the cue-rotation and object displacement condition and the cue-replacement and object displacement condition compared to the object displacement only condition particularly towards the non-displaced objects. If overshadowing of the distal cues by the proximal cues were to occur we would suggest that no differences between the conditions would be observed.

\subsection{Method}

3.1.1. Animals

Male Wistar rats (200-300 g) were again used in this study. The housing and handling conditions were identical to those in the first study.

\subsubsection{Apparatus}

The testing arena used in Study 1 was again employed here.

\subsubsection{Procedure}

All rats were placed in the centre of the arena and were given four trials for a single day to explore the arena. A single day of training was chosen because the results of Study 1 would indicate that habituation occurs mainly on the first day, with no significant decrease in reactivity between days 2, 3 or 4. Similar to Study 1 each trial lasted $1 \mathrm{~min}$ and the inter-trial interval was approximately $15 \mathrm{~s}$. To assess exploratory behaviour the experimenter again recorded the number of nose contacts each rat made with the individual objects.

Retention was assessed 7 days post-habituation as in the first experiment. Animals were then divided into two groups for both the distal cue rotation experiment and the distal cue replacement experiment.

\subsubsection{Distal cue rotation experiment. The object} displacement group $(n=7)$ was placed in the centre of the arena for $1 \mathrm{~min}$ where the wooden object was moved toward the side of the arena but none of the distal visual cues were changed.

The distal cue rotation and object displacement group $(n=8)$ was also placed for $1 \mathrm{~min}$ in the arena where the cues were rotated $180^{\circ}$ clockwise. In addition, the wooden block was moved toward the side of the arena.

3.1.3.2. Distal cue replacement experiment. The object displacement group $(n=7)$ was placed in the centre of the arena for $1 \mathrm{~min}$ where the wooden object was moved toward the side of the arena but none of the distal visual cues were changed.

The distal cue replacement and object displacement group $(n=8)$ was also placed for $1 \mathrm{~min}$ in the arena where the wooden block was moved toward the side of the arena. In addition, the black poster that was suspended on the eastern wall was replaced with a black 
and white striped poster of similar dimensions. The white laboratory coat worn by the experimenter at the southern position was replaced with a dark coloured jumper. The two coloured bulbs located in the northeast and northwest positions were replaced with two white $(60 \mathrm{~W})$ bulbs.

\subsubsection{Statistics}

A series of repeated and/or mixed ANOVAs were used. Where appropriate independent and/or dependent $t$-tests were carried out using SPSS (Version 10) $\left({ }^{*} P<0.05 ;{ }^{* *} P<0.01\right)$. In order to directly compare the object displacement groups with both the cuerotation and object displacement group and the cuereplacement and object displacement group all data was normalised to percentage increase in exploration.
A score of $100 \%$ indicated that there was no increase in exploration on the retention trial when compared to the final trial of the habituation period.

\subsection{Results}

\subsubsection{Distal cue rotation experiment}

All animals habituated to the arena over the four trials. The mean number of nose contacts made with all objects in the arena decreased from $26.3 \pm 2.05$ on the first trial to $8.13 \pm 1.66$ on trial 4 (see Fig. 3a). This habituation was confirmed by a one-way ANOVA. An overall significant difference was found between the four trials $(F=14.85$, d.f. $=3,56, P<0.01)$. Subsequent post-hoc analysis (Tukey $P<0.05$ ) revealed that the number of nose contacts made with the objects on trial

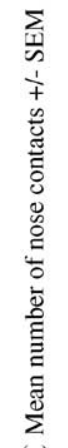

(a)
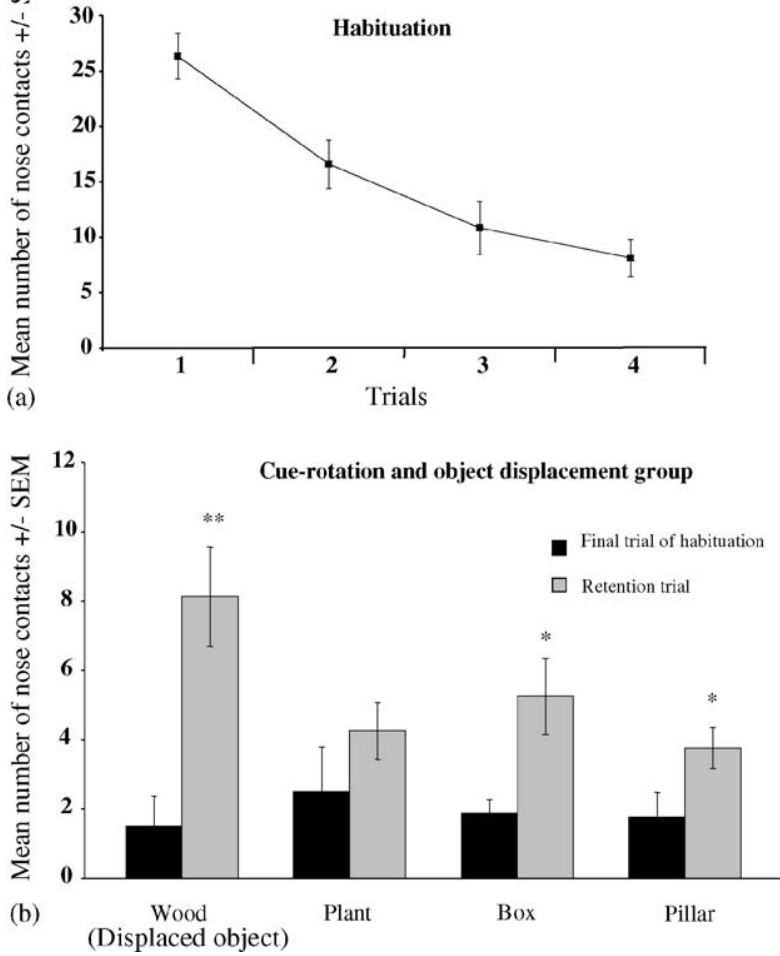

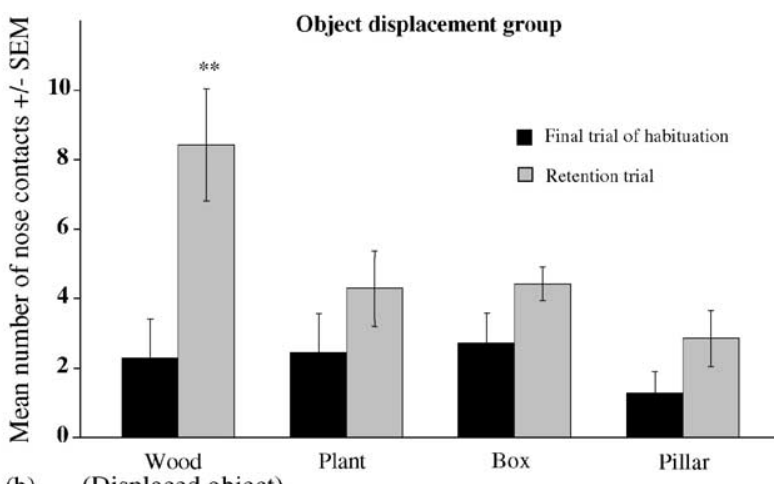

(b) (Displaced object)

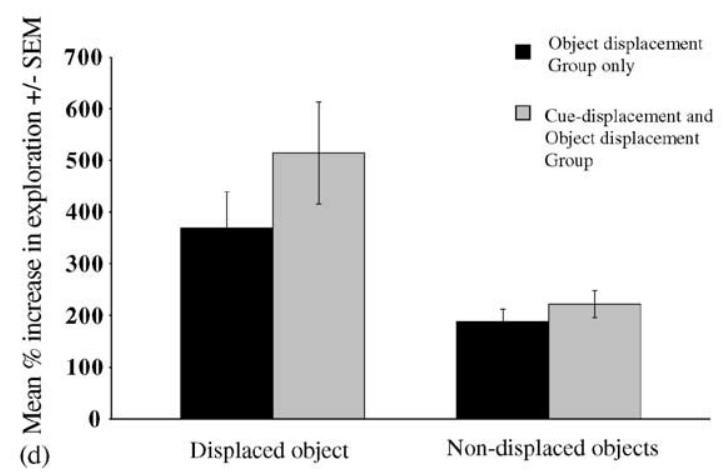

Fig. 3. (a) The mean number of nose contacts made with all objects over the four trials of habituation for the cue-rotation experiment. (b) and (c) the mean number of nose contacts made with each of the four objects on the last trial of the last day of habituation compared to the retention trial for the object displacement group (b) and the cue-rotation and object displacement group (c). (d) The direct comparison of the mean percentage increases in exploration of either the displaced object or the non-displaced objects on the retention trial (compared to the final trial of habituation) for both the object displacement group and the cue-rotation and object displacement groups. 
4 was significantly less than on trials 1 and 2 . Similarly, the number of nose contacts made with the objects on trial 3 was also significantly lower than that on the first trial.

Reaction to distal cue rotation was assessed 7 days post-habituation. We first compared the object displacement only group on their mean number of nose contacts made with each individual object on the retention trial to the mean number of nose contacts on the final trial of habituation. A repeatedmeasures ANOVA with object type (wood, plant, box and pillar) as the between-group measure and trial (final trial versus retention trial) as the within group measures was conducted. This revealed an overall significant effect for trial $(F=19.7$, d.f. $=1,24$, $P<0.01)$. There was also a significant effect for object $(F=4.88$, d.f. $=3,24, P<0.05)$ and a significant trial X object interaction effect $(F=4.39$, d.f. $=3,24$, $P<0.05)$. Subsequent $t$-tests revealed that the number of nose contacts made with the wood (displaced object) significantly increased (Fig. $3 b, t=-5.19$, d.f. $=6$, $P<0.01)$ on the retention trial when compared to the final trial of habituation. No other differences were noted.

A further repeated ANOVA was conducted for the cue-rotation and object displacement group. Object type was again used as the between-group measure and trial as the within-group measure. This revealed a significant effect for trial $(F=28.37$, d.f. $=1,28$, $P<0.01)$. There was a non-significant effect for object type $(F=1.49$, d.f. $=3,28, P>0.05)$. But a significant object $\mathrm{X}$ trial interaction effect was found $(F=3.02$, d.f. $=3,28, P<0.05)$. Subsequent $t$-test analyses revealed that that the number of nose contacts made with the wood (displaced object, $t=-4.08$, d.f. $=7$, $P<0.01)$, box $(t=-3.21$, d.f. $=7, P<0.05)$ and pillar $(t=-2.57$, d.f. $=7, P<0.05)$ had significantly increased on the retention trial when compared to the final trial of habituation (see Fig. 3c).

To directly examine whether the cue-rotation and object displacement group reacted more to any or all of the objects compared to the object displacement only group on the retention trial a further ANOVA was conducted. Group (object displacement group versus object displacement and cue-rotation group) was one between group measure and object (displaced object versus non-displaced objects) was the second. This revealed a significant effect for group
$(F=4.95$, d.f. $=1,56, P<0.05)$ and a significant effect for object $(F=29.06$, d.f. $=1,56, P<0.01)$. In addition, there was a non-significant group $\mathrm{X}$ object interaction effect $(F=2.22$, d.f. $=1,56 P>0.05)$. This would suggest that the cue-displacement group did not display any additional exploration to either the displaced object or the non-displaced objects when compared to the object displacement group alone (see Fig. 3d).

\subsubsection{Distal cue replacement experiment}

All animals habituated to the arena over the four trials. The mean number of nose contacts made with all objects in the arena decreased from $33.6 \pm 1.94$ on trial 1 to $7.46 \pm 1.95$ on trial 4 (see Fig. 4a). This habituation was confirmed by a one-way ANOVA. An overall significant difference was found between the four trials $(F=39.36$, d.f. $=3,56, P<0.01)$. Subsequent post-hoc analysis (Tukey $P<0.05$ ) revealed that the number of nose contacts made with the objects on trial 4 was significantly less than on trials 1 and 2. Similarly, the number of nose contacts made with the objects on trial 3 was also significantly lower than that on the first trial and second trial. While the mean number of nose contacts on trial 2 was significantly lower than that on trial 1 .

Reaction to distal cue replacement was assessed 7 days post-habituation. We first compared the object displacement only group on their mean number of nose contacts made with each individual object on the retention trial to the mean number of nose contacts on the final trial of habituation. A repeated-measures ANOVA with object type (wood, plant, box and pillar) as the between-group measure and trial (final trial versus retention trial) as the within group measures was conducted. This revealed an overall significant effect for trial $(F=72.17$, d.f. $=1,24, P<0.01)$. There was also a significant effect for object $(F=21.2$, d.f. $=3$, $24, P<0.01)$ and a significant trial $\mathrm{X}$ object interaction effect $(F=12.63$, d.f. $=3,24, P<0.01)$. Subsequent $t$-test analyses revealed that that the number of nose contacts made with the wood (displaced object, $t=-5.75$, d.f. $=6, P<0.01)$, plant $(t=-4.6$, d.f. $=6, P<0.01)$, box $(t=-3.58$, d.f. $=6, P<0.01)$ and pillar $(t=-3.65$, d.f. $=6, P<0.01)$ had significantly increased on the retention trial when compared to the respective final trial of habituation (see Fig. 4b). 

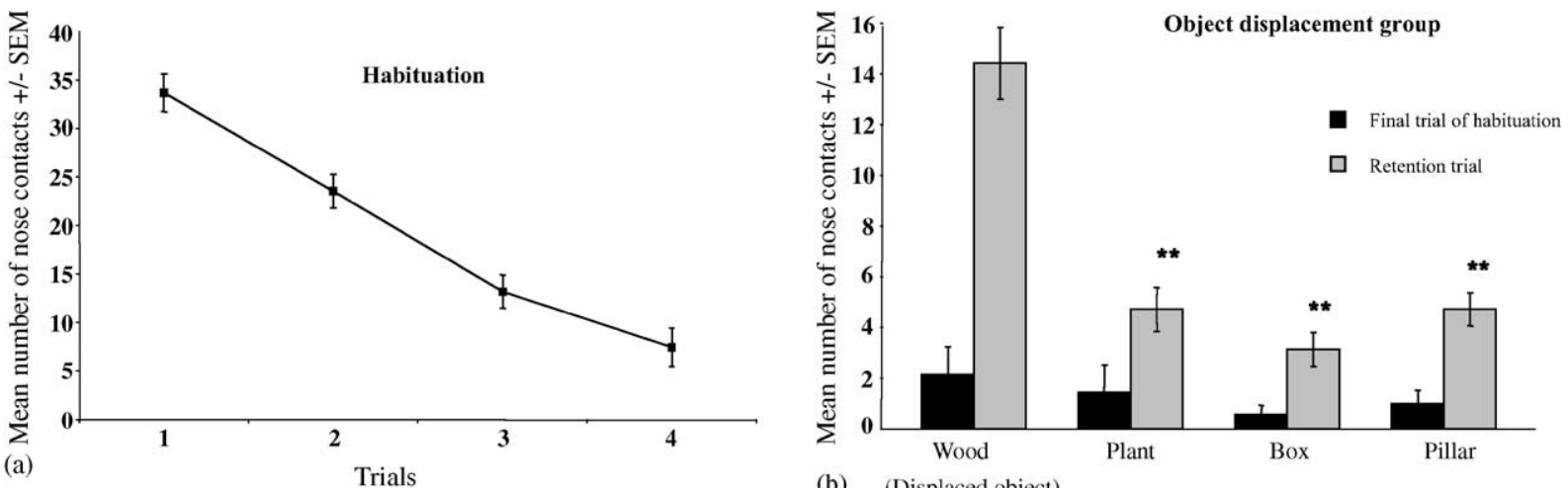

(b) (Displaced object)
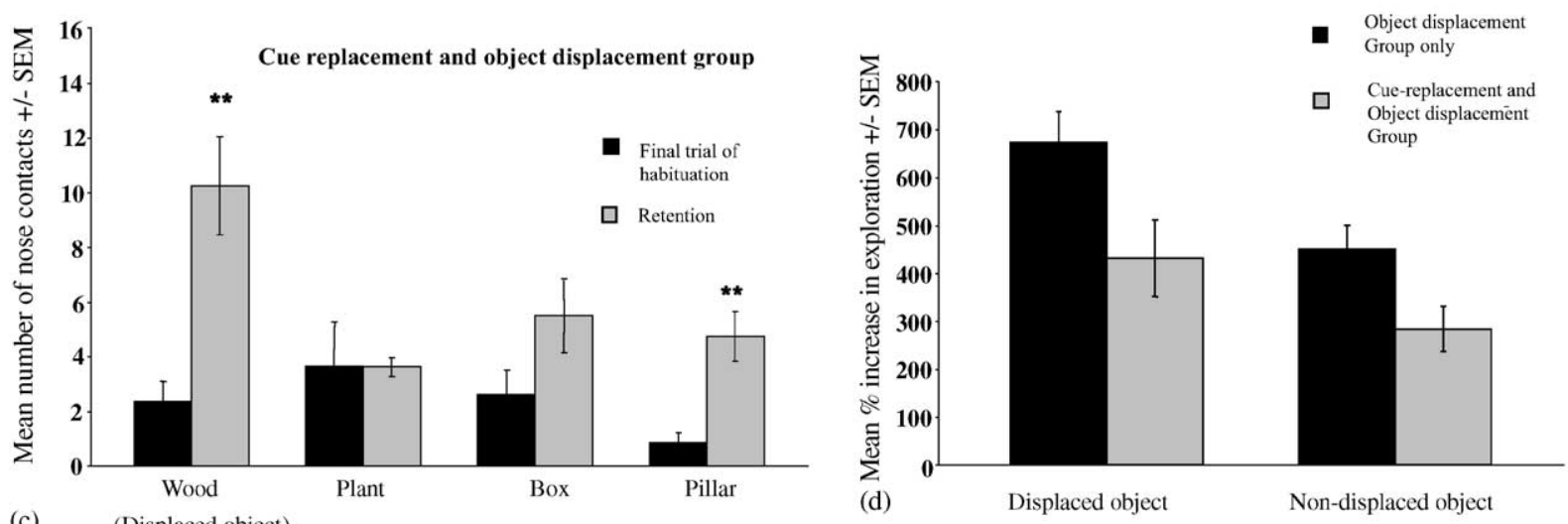

Fig. 4. (a) The mean number of nose contacts made with all objects over the four trials of habituation for the cue-replacement experiment. (b) and (c) the mean number of nose contacts made with each of the four objects on the last trial of the last day of habituation compared to the retention trial for the object displacement group (b) and the cue-replacement and object displacement group (c). (d) A direct comparison of the mean the mean percentage increase in exploration of either the displaced object or the non-displaced objects on the retention trial (compared to the final trial of habituation) for both the object displacement group and the cue-replacement and object displacement group.

A further repeated ANOVA was conducted for the cue-replacement and object displacement group. Object type was again used as the between-group measure and trial as the within-group measure. This revealed a significant effect for trial $(F=21.91$, d.f. $=1,28, P<0.01)$. There was also a significant effect for object type $(F=3.22$, d.f. $=3,28, P<0.05)$. A significant object $\mathrm{X}$ trial interaction effect was found $(F=4.35$, d.f. $=3,28, P<0.05)$. Subsequent $t$-test analyses revealed that that the number of nose contacts made with the wood (displaced object, $t=-4.27$, d.f. $=7, P<0.01)$ and pillar $(t=-5.4$, d.f. $=7, P<0.05)$ had significantly increased on the retention trial when compared to the final trial of habituation (see Fig. 4c).

To directly examine whether the cue-replacement and object displacement group reacted more to any or all of the objects compared to the object displacement only group on the retention trial a further ANOVA was conducted. Group (object displacement versus object displacement and cue-replacement) was one between group measure and object (displaced object versus non-displaced objects) was the second. This revealed a significant effect for group $(F=8.14$, d.f. $=1,56, P<0.01)$. There was also a significant effect for object $(F=6.7$, d.f. $=1,56, P<0.01)$. In addition, there was a non-significant group $\mathrm{X}$ object interaction effect $(F=0.28$, d.f. $=1,56, P>0.05)$. This would suggest that the cue-replacement group did not display any additional exploration to either the displaced object or the non-displaced objects when compared to the object displacement group alone (see Fig. 4d). 


\subsection{Discussion}

Displacement of an object in all conditions resulted in a general increase in exploration and a significant increase around the particular object that was displaced. This demonstrates that animals can passively acquire spatial knowledge of an environment rapidly (over four trials, as suggested by Study 1) and that this knowledge is retained for at least 7 days. More importantly, results from both the cue-displacement and the cue-replacement experiments would indicate that when compared directly to the displaced object only condition no extra increase in exploration to either the displaced or non-displaced objects occurred. This perhaps would suggest that during the retention trial the distal cues were relatively ignored and only the proximal change had been noticed in both experiments. In both the cue-displacement and the cue-replacement conditions, we observed a significant increase in exploration around the displaced object itself (similar to the object displacement only condition) again suggesting an overshadowing of extramaze cues by intramaze ones. To our knowledge this is the first experiment to demonstrate such an overshadowing effect in a passive learning task. In contrast, rats in a more active learning task such as the radial-arm maze tend to rely on distal allocentric cues (Hunt and Aggleton, 1998; Neave et al., 1997) and will show an overshadowing of intramaze cues by extramaze ones (Diez-Chamizo et al., 1985). In the object exploration task, the objects are in the immediate vicinity of the animal. It is possible that the animals would have a greater awareness of any disruption in the immediate arena in comparison to a disruption in distally placed cues solely as a result of their poor vision (Robinson et al., 2001). Alternatively as this is a passive learning task the distal visual cues have no reward value, they do not predict the location of food etc and therefore they can be relatively ignored. Biegler and Morris (1996) suggest that spatial representations are organised hierarchically, with proximal landmarks being treated as more accurate while distal features are considered more reliable but again this is in the context of obtaining a reward. They further suggest that if a conflict arises after training in a stable environment, the weight given to each source of spatial information will depend on its previous reliability and extent of the discrepancy (Biegler and Morris, 1996). We add to this by suggesting that weight given to either the proximal cues or distal cues will also depend on the predictive reward value of each. In the context where neither type of cue predicts a reward, the cues most proximally located will be given more weight and attention. Future studies should examine comparisons relevant to the reward value of proximal and distal cues are made in an object displacement task.

\section{Conclusions}

In conclusion, the experiments presented here demonstrate that both proximal and distal cues are retained in long-term spatial memory following a task that has been passively acquired. We also suggest that this spatial knowledge is acquired rapidly (over four trials) and retained for at least a week therefore confirming our previous findings (Commins et al., 2003). In addition, we demonstrate that when a conflict arises between distal and proximal cues, the disruption in proximal cues overshadows the disruption in distal cues.

\section{Acknowledgements}

This work was supported by the Health Research Board of Ireland and the Department of Psychology, NUI Maynooth.

\section{References}

Biegler, R., Morris, R.G.M., 1996. Landmark stability: studies exploring whether the perceived stability of the environment influences spatial representations. J. Exp. Biol. 199, 187-193.

Bolhuis, J.J., Stewart, C.A., Forrest, E.M., 1994. Retrograde amnesia and memory reactivation in rats with ibotenate lesions to the hippocampus or subiculum. Q. J. Exp. Psychol. B. 47, 129-150.

Carman, H.M., Mactutus, C.F., 2002. Proximal versus distal cue utilization in spatial navigation: the role of visual acuity? Neurobiol. Learn. Mem. 78 (2), 332-346.

Chapillion, P., Roullet, P., Lassalle, J.M., 1995. Ontogeny of orientation and spatial learning on the radial maze in mice. Dev. Psychobiol. 28 (8), 429-442.

Chapillion, P., 1999. Very brief exposure to visual distal cues is sufficient for young mice to navigate in the Morris water maze. Behav. Proc. 46, 15-24.

Chapius, N., Durup, M., Thinus-Blanc, C., 1987. The role of exploratory experience in a shortcut task by golden hamsters. Anim. Learn. Behav. 15, 174-178. 
Commins, S., Cunningham, L., Harvey, D., Walsh, D., 2003. Massed but not spaced training impairs spatial memory. Behav. Brain Res. 139, 215-223.

Dai, H., Krest, M., Carey, R.J., 1995. A new methodological approach to the study of habituation: the use of positive and negative behavioural indices of habituation. J. Neurosci. Methods 62, 169-174.

Delu, F., Fauchey, V., Le Moal, M., Simon, H., 1997. Extension of a new two-trial memory task in the rat: influence of environmental context on recognition processes. Neurobiol. Learn. Mem. 67 (2), $112-120$.

Diez-Chamizo, V., Sterio, D., Mackintosh, N.J., 1985. Blocking and over-shadowing between intramaze and extramaze cues-a test of the independence of locale and guidance learning. Q. J. Exp. Psychol. 37B (3), 235-253.

Dix, S.L., Aggleton, J.P., 1999. Extending the spontaneous preference test of recognition: evidence of object-location and objectcontext recognition. Behav. Brain Res. 99, 191-200.

Eilam, D., Golani, I., 1989. Home-base behaviour of rats Rattus norvegicus exploring a novel environment. Behav. Brain Res. 34, 199-211.

Ellen, P., Parke, E.M., Wages, C., Doherty, P., Hermann, T., 1982. Spatial problem solving by rats: exploration and cognitive maps. Learn. Motiv. 13, 81-94.

Ellen, P., Soteres, B.J., Wages, C., 1984. Problem solving in the rat: piecemeal acquisition of cognitive maps. Anim. Learn. Behav. 12, 232-237.

Ennaceur, A., Delacour, J., 1988. A new one-trial test for neurobiological studies of memory in rats 1: behavioural data. Behav. Brain Res. 31 (1), 47-59.

Galani, R., Weiss, I., Cassel, J.C., Kelche, C., 1998. Spatial memory, habituation and reactions to spatial and nonspatial changes in rats with selective lesions of the hippocampus, the entorhinal cortex or the subiculum. Behav. Brain Res. 96, 1-12.

Gemmell, C., O’Mara, S.M., 1999. Medial prefrontal cortex lesions cause deficits in a variable-goal location task but not in object exploration. Behav. Neurosci. 113 (3), 465-474.

Granon, S., Save, E., Buhot, M.C., 1996. Effortful information processing in a spontaneous spatial situation by rats with medial prefrontal lesion. Behav. Brain Res. 78, 147-154.

Hunt, P.R., Aggleton, J.P., 1998. An examination of the spatial working memory deficit following neurotoxic medial dorsal thalamic lesions in rats. Behav. Brain Res. 97, 129-141.

Mc Gauran, A.T., Harvey, D., Cunningham, L., Craig, S., Commins, S., 2004. Retention of cue-based associations in the water maze is time-dependent and sensitive to disruption by rotating the starting position. Behav. Brain Res. 151, 255-266.
Moses, S.N., Sutherland, R.J., Mc Donald, R.J., 2002. Differential involvement of amygdala and hippocampus in responding to novel objects and contexts. Brain Res. Bull. 58 (5), 517527.

Mumby, D.G., Cameli, L., Glenn, M.J., 1999. Impaired allocentric spatial working memory and intact retrograde memory after thalamic damage caused by thiamine deficiency in rats. Behav. Neurosci. $113(1), 42-50$.

Neave, N., Nagle, S., Aggleton, J.P., 1997. Evidence for the involvement of the mamillary bodies and cingulum bundle in allocentric spatial processing by rats. Eur. J. Neuro. 9, 941-955.

O’Keefe, J., Nadel, L., 1978. The Hippocampus as a Cognitive Map. Oxford University Press, London.

Poucet, B., 1989. Object exploration, habituation and response to a spatial change in rats following septal or medial frontal cortical damage. Behav. Neurosci. 103 (5), 1009-1016.

Renner, M.J., 1988. Learning during exploration: the role of behavioural topography during exploration in determining subsequent adaptive behaviour. Int. J. Comp. Psychol. 2, 43-56.

Renner, M.J., 1990. Neglected aspects of exploratory and investigatory behaviour. Psychobiology 18, 16-22.

Robinson, L., Bridge, H., Riedel, G., 2001. Visual discrimination learning in the water maze: a novel test for visual acuity. Behav. Brain Res. 119, 77-84.

Rudy, J.W., Stadler-Morris, S., Albert, P., 1987. Ontogeny of spatial navigation behaviours in the rat: dissociation of "proximal"and "distal"-cue-based behaviours. Behav. Neurosci. 101 (1), 62-73.

Save, E., Poucet, B., Foreman, N., Buhot, M.C., 1992. Object exploration and reactions to spatial and nonspatial changes in hooded rats following damage to parietal cortex or hipocampal formation. Behav. Neurosci. 106, 447-456.

Save, E., Poucet, B., 2000. Involvement of the hippocampus and associative parietal cortex in the use of proximal and distal landmarks for navigation. Behav. Brain Res. 109, 195-206.

Tropp, J., Markus, E.J., 2001. Sex differences in the dynamics of cue utilization and exploratory behaviour. Behav. Brain Res. 119, 143-154.

Thinus-Blanc, C., Durup, M., Poucet, B., 1992. The spatial parameters encoded by hamsters during exploration: a further study. Behav. Proc. 26 (1), 43-57.

Van Groen, T., Kadish, I., Wyss, J.M., 2001. Old rats remember old tricks: memories of the water maze persist for 12 months. Behav. Brain Res. 136, 247-255.

Vollmer-Conna, U.S., Lemon, J., 1998. Spatial configuration and proximal cues. Learn. Motiv. 29 (1), 102-111. 